\title{
Sulfur Hexafluoride Lipid Microspheres
}

National Cancer Institute

\section{Source}

National Cancer Institute. Sulfur Hexafluoride Lipid Microspheres. NCI Thesaurus. Code C138075.

A contrast agent composed of lipid microspheres containing an inorg anic fluorinated inert gas comprised of six fluoride atoms bound to one sulfur atom (SF6), with potential contrast enhancing activity for diagnostic imaging during ultrasound procedures. Upon administration of the sulfur hexafluoride lipid microspheres, the microspheres are distributed throughout the bloodstream and into organs. The acoustic impedance of the lipid microspheres is much lower than that of the surrounding tissue and/or fluids. Therefore during ultrasound imaging, this agent reflects the ultrasound waves at the microsphere-tissue interface, which enhances the contrast between the areas where the microspheres are present and the surrounding tissues or fluids. 